Kohlenhydrate, Flüssigkeit, Blutzuckermessgerät

\title{
Das gehört in die Sporttasche eines Diabetikers
}

- Chronische Erkrankungen wie Diabetes sind kein Hindernis, Sport zu treiben. „Insbesondere für Menschen mit Typ-2-Diabetes ist regelmäßige Bewegung, vor allem Ausdauersport, wichtig", erklärt Prof. Ingo Froböse, Köln. Nicht selten können dadurch Medikamente reduziert oder ausgesetzt werden.

Die Deutsche Diabetes Gesellschaft (DDG) empfiehlt, die anfängliche Belastungsdauer von zehn Minuten nicht zu überschreiten und diese über Wochen langsam zu steigern. Um bei Typ-2-Diabetes langfristige Erfolge wie eine Blutzuckersenkung zu erzielen, empfiehlt die DDG Belastungsintervalle von 30 bis 60 Min. drei- bis viermal wöchentlich.

Das Training sollte genau geplant werden. „Neben ,Not-Kohlenhydraten' und genügend Flüssigkeit muss auch ein Blutzuckermessgerät seinen festen Platz in der Sporttasche haben", verdeutlicht Ulla Gatzweiler, Lauftrainerin beim Diabetes Pro- gramm Deutschland. Die Blutzuckerbestimmung durch ein präzises, leicht zu bedienendes Messgerät wie CONTOUR $^{\circledR}$ XT ist dabei entscheidend.

\section{Regelmäßige Blutzuckermessung während des Trainings wichtig}

Froböse hebt die Bedeutung einer genauen Blutzuckermessung beim Sport hervor: „Durch die gesteigerte Insulinempfindlichkeit der Muskelzellen sinkt der Blutzuckerspiegel beim Sport ab. Gleichzeitig benötigt der Körper mehr Energie. Um in der Folge eine Unterzuckerung zu vermeiden, ist es notwendig, die Dosis an benötigtem Insulin anzupassen oder Kohlenhydrate zu sich zu nehmen. Mit Hilfe regelmäßiger Blutzuckerselbstkontrollen während des Trainings können diese Entscheidungen leichter getroffen werden."

\footnotetext{
- Red.

Quelle: Nach Presseinformationen von Bayer HealthCare
}

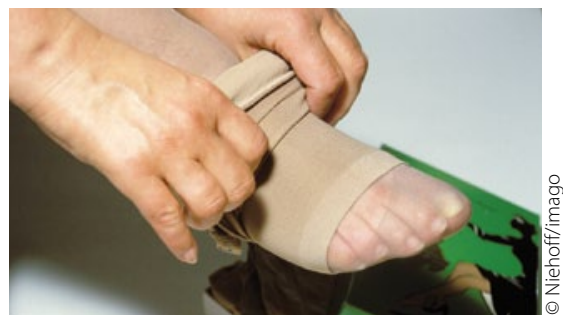

Thrombose-Strümpfe sollten Qualitätskritierien erfüllen.

\section{Thromboseprophylaxe}

\section{Medikamentös und/ oder physikalisch?}

— In der Praxis stellt sich oft die Frage, wie eine individuelle und risikoadaptierte Prophylaxe venöser Thromboembolien (VTE) umgesetzt werden sollte: medikamentös, mit physikalischen Methoden oder mit beiden Strategien.

Einig waren sich die Experten*, dass medizinische Thromboseprophylaxestrümpfe (MTPS) entsprechend der S3-Leitlinie „Prophylaxe der VTE“ (www.awmf.org/ uploads/tx_szleitlinien/003-001I_S3_ Thromboembolie-Prophylaxe_2010.pdf) u. a. bei Patienten mit mittlerem bis hohem VTE-Risiko und Kontraindikationen gegen Antikoagulanzien angewendet werden sollten. Prof. Knut Kröger, Krefeld, forderte darüber hinaus, die Indikationen, bei denen MTPS bzw. MTPS in Kombination mit Heparinen eingesetzt werden sollen, präziser zu definieren. „Geprüft werden sollte auch, ob es Patienten gibt, deren VTE-Risiko so gering ist, dass sie entweder nur MTPS oder möglicherweise auch gar keine Prophylaxe benötigen", so Kröger.

Prof. Peter Kujath, Lübeck, unterstrich, dass MTPS einen in Studien nachgewiesenen antithrombotischen Effekt haben. „Allerdings unterscheiden sich die einzelnen Fabrikate im Hinblick auf ihre biomechanischen Eigenschaften zum Teil erheblich voneinander", so Kujath. Eine aktuelle Untersuchung der TU Berlin zeigte, „dass nicht alle Modelle den für MTPS geforderten kontinuierlichen Druckabfall von distal nach proximal gewährleisteten", ergänzte Kujath.

- Red.

Quelle: *Expertengespräch, Köln, Juli 2012

(Veranstalter: Medical Data Institute)
Quelle: Satellitensymposium „Advancing TAVI and Saving Lives - 10 years after the first implant", DGK-Jahrestagung, Mannheim, April 2012 (Veranstalter: Edwards Lifescience) 